\title{
DOUBLY EXPONENTIALLY MANY INGLETON MATROIDS
}

\author{
PETER NELSON AND JORN VAN DER POL
}

\begin{abstract}
A matroid is Ingleton if all quadruples of subsets of its ground set satisfy Ingleton's inequality. In particular, representable matroids are Ingleton. We show that the number of Ingleton matroids on ground set $[n]$ is doubly exponential in $n$; it follows that almost all Ingleton matroids are non-representable.
\end{abstract}

\section{INTRODUCTION}

Ingleton's condition Ing71 is a well-known linear inequality that holds for representable matroids but not matroids in general; it states that for all $A, B, C, D \subseteq E$ in a representable matroid $M=(E, r)$,

$$
\begin{aligned}
& r(A \cup B)+r(A \cup C)+r(A \cup D)+r(B \cup C)+r(B \cup D) \\
& \quad \geq r(A)+r(B)+r(A \cup B \cup C)+r(A \cup B \cup D)+r(C \cup D) .
\end{aligned}
$$

An arbitrary matroid is Ingleton if the above inequality is satisfied for all choices of $A, B, C, D$. The class of Ingleton matroids is closed under minors and duality (see, for example, Lemmas 3.9 and 4.5 in [Cam13]) and clearly all representable matroids are Ingleton. A natural question is to what extent a converse of this last statement holds: that is, do Ingleton matroids tend to be representable? We prove here that they do not. For $n \geq 12$, the number of representable matroids on $[n]$ is at most $2^{0.25 n^{3}}$ NNel16; our main result is the following.

Theorem 1.1. For all sufficiently large $n$ and all $0<r<n$, the number of Ingleton matroids with ground set $[n]$ is at least $2^{0.486 \frac{\log (r(n-r))}{r(n-r)}\left(\begin{array}{l}n \\ r\end{array}\right)}$.

Even when $r=4$, this eclipses the upper bound on the number of representable matroids on $[n]$ with no restriction on rank; thus, almost all Ingleton matroids are non-representable. When $r=\lfloor n / 2\rfloor$, the above formula is around $2^{\frac{1.94}{n^{2}}\left(\begin{array}{c}n \\ n / 2\end{array}\right)}$ which is doubly exponential in $n$,

The first author was supported by a grant from the Natural Sciences and Engineering Research Council of Canada.

The second author was supported by the Netherlands Organisation for Scientific Research (NWO) grant 613.001.211. 
and even somewhat resembles the number of all matroids on $[n]$, which is $2^{\Theta\left(\frac{1}{n}\left(\begin{array}{c}n \\ \lfloor n / 2\rfloor\end{array}\right)\right)}$ with a constant between 1 and $2+o(1)$ BPvdP15. We conjecture, however, that general matroids tend not to be Ingleton:

Conjecture 1.2. There is a constant c such that the number of Ingle-

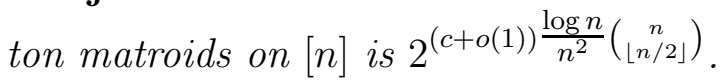

Conceivably, the constant $c$ could be equal to 2 , or even be the one of around 1.94 obtained by our proof.

In what follows, we assume some familiarity with matroid theory; see Oxl11. In particular, a nonbasis of a rank- $r$ matroid $M$ is a set in $\left(\begin{array}{c}E(M) \\ r\end{array}\right)$ that is not a basis of $M$; and a matroid is paving if all its nonbases are circuits and sparse paving if $M$ and $M^{*}$ are both paving. Logarithms are all base-two.

As part of the proof of Theorem 1.1, we also characterize exactly which sparse paving matroids are Ingleton, and as a result easily derive the following theorem:

Theorem 1.3. There are exactly 41 excluded minors for the class of Ingleton sparse paving matroids: the matroids $U_{0,2} \oplus U_{1,1}$ and $U_{2,2} \oplus$ $U_{0,1}$, and the 39 rank-4 non-Ingleton sparse paving matroids on eight elements.

The fact that this set is even finite is curious; the class of all Ingleton matroids, by contrast, has an infinite set of excluded minors, constructed in [MNW09]. In fact, their techniques show that every $\mathbb{R}$-representable matroid is a minor of an excluded minor for the class of Ingleton matroids.

Theorems 1.1 and 1.3 together imply that the Ingleton matroids are a 'large' minor-closed class of matroids (in the sense of numbering at least $2^{2^{n}} / \operatorname{poly}(n)$ that omits 39 different sparse paving matroids. It was conjectured in [MNWW11] that any minor-closed class not containing all sparse paving matroids is asymptotically vanishing; our result shows that such a class may still be 'large'.

\section{REPRESENTING MATROIDS WITH FEW NONBASES}

Lemma 2.1. Let $M$ be a rank-r matroid in which each set $\mathcal{W}$ of nonbases with $|\mathcal{W}|>1$ satisfies $|\cap \mathcal{W}| \leq r-|\mathcal{W}|$. Then $M$ is $\mathbb{R}$ representable.

Proof. Let $\mathcal{X}=\left\{X_{1}, \ldots, X_{t}\right\}$ be the set of nonbases of $M$; note that $0 \leq|\cap \mathcal{X}| \leq r-t$, so $t \leq r$. Let $A$ be an $[r] \times E$ real matrix so that the nonzero entries of $A$ are algebraically independent over $\mathbb{Q}$, and $A_{i, e}=0$ 
if and only if $i \in[t]$ and $e \in X_{i}$. We prove that $M=M(A)$. It is clear that for every nonbasis $W$ of $M$, the matrix $A[W]$ has a zero row so is singular; it remains to show that $A[B]$ is nonsingular for each basis $B$ of $M$.

Let $B$ be a basis of $M$. Consider the bipartite graph $G$ with bipartition $([r], B)$ for which $(i, e)$ is an edge if and only if $A_{i, e} \neq 0$. Note that each $i \in\{t+1, \ldots, r\}$ has degree $r$ in $G$. For each $S \subseteq[r]$, let $N(S)$ denote the set of vertices in $B$ that are adjacent to a vertex in $S$. We argue that $|N(S)| \geq|S|$ for each $S \subseteq[r]$; it will follow from Hall's theorem that $G$ has a perfect matching.

Let $S \subseteq[r]$. If $S \nsubseteq[t]$ then clearly $N(S)=B$ and so $|N(S)|=r \geq$ $|S|$. If $S \subseteq[t]$, then by hypothesis the set $\bigcap_{s \in S} X_{s}$ has size at most $r-|S|$, so $B$ contains at least $|S|$ elements $e$ for which there is some $s \in S$ with $e \notin X_{s}$. Each such $e$ is adjacent to $s$, so $|N(S)| \geq|S|$ as required. Therefore $G$ has a perfect matching.

Let $B=\left\{b_{1}, \ldots, b_{r}\right\}$ and $S_{r}$ denote the symmetric group on $[r]$; note that $A[B]$ is singular if and only if the determinant $\sum_{\sigma \in S_{r}} \prod_{i \in[r]} A_{i, b_{\sigma(i)}}$ is zero. This determinant is a polynomial in the entries of $A$, with integer coefficients, whose nonzero monomials are algebraically independent over $\mathbb{Q}$, and since $G$ has a perfect matching, some monomial is nonzero. It follows that the determinant is nonzero, so $A[B]$ is nonsingular, as required.

Lemma 2.2. Every matroid with at most four nonbases is $\mathbb{R}$ representable.

Proof. Let $M$ be a minor-minimal counterexample. Note that $M$ is simple, that $r(M) \geq 3$, and that $M^{*}$ is also a minor-minimal counterexample, so $M$ is cosimple with $r^{*}(M) \geq 3$. If $M$ has an element $e$ in no nonbases, then since $e$ is not a coloop, $M$ is the free extension of $M \backslash e$ by $e$; since $M \backslash e$ is $\mathbb{R}$-representable, so is $M$, a contradiction. So every element is in a nonbasis of $M$. Dually, no element is in all nonbases of $M$.

If $M$ has a dependent set $Y$ of size $r(M)-1$, then $Y \cup\{e\}$ is a nonbasis for each $e \in E(M)-Y$. Since no element of $Y$ is in four nonbases, this gives $|E(M)-Y| \leq 3$, so $|E(M)| \leq r+2$, giving $r^{*}(M) \leq 2$, a contradiction. Therefore every circuit of $M$ is spanning, so $M$ is a paving matroid; dually, $M$ is a sparse paving matroid.

If $e \in E(M)$ is in exactly one nonbasis $X$, then $M$ is the principal extension of the flat $X-\{e\}$ in $M \backslash e$, so $M$ is $\mathbb{R}$-representable, a contradiction. Therefore every $e \in E(M)$ is in at least two nonbases. Dually, every element lies outside at least two nonbases. Therefore $M$ has exactly four nonbases, and every element is in exactly two of them. 
If $r(M)=3$, then $M$ has four triangles, so there are 12 pairs $(e, T)$ where $T$ is a triangle containing $e$, and every element is in two triangles, so there are also $2|E(M)|$ such pairs $(e, T)$. Thus $|E(M)|=6$ and so $M$ is $\mathbb{R}$-representable, a contradiction.

Suppose, therefore, that $r(M) \geq 4$. By Lemma 2.1, we may assume that there is some set $\mathcal{X}$ of nonbases of $M$ with $|\mathcal{X}|>1$ such that $|\cap \mathcal{X}|>r-|\mathcal{X}|$. Since no element is in three nonbases, if $|\mathcal{X}|>2$ then $|\cap \mathcal{X}|=0 \leq r-|\mathcal{X}|$, so we must have $|\mathcal{X}|=2$ and thus there are nonbases $X_{1}, X_{2}$ with $\left|X_{1} \cap X_{2}\right|=r-1$. This contradicts the fact that $M$ is a sparse paving matroid.

\section{Ingleton Matroids}

In this section, we use the well-known fact that $H \subseteq\left(\begin{array}{l}E \\ r\end{array}\right)$ is the set of nonbases of a sparse paving matroid on $E$ if and only if no two elements of $H$ have intersection of size exactly $r-1$.

Lemma 3.1. Let $M$ be a rank-r sparse paving matroid. Sets $A, B, C, D$ violate the Ingleton inequality in $M$ if and only if there are pairwise disjoint sets $X_{1}, X_{2}, X_{3}, X_{4}, Y, Z_{1}, Z_{2} \subseteq E(M)$ such that

- $\left|X_{i}\right|=2$ for each $i \in[4]$ while $\left|Y \cup Z_{1} \cup Z_{2}\right|=r-4$,

- $A=X_{1} \cup Y \cup Z_{1} \cup Z_{2}$,

- $B=X_{2} \cup Y \cup Z_{1} \cup Z_{2}$,

- $C=X_{3} \cup Y \cup Z_{1}$, and

- $D=X_{4} \cup Y \cup Z_{2}$,

while each of $A \cup B, A \cup C, A \cup D, B \cup C$ and $B \cup D$ is a circuit-hyperplane of $M$, and $C \cup D$ is a basis.

Proof. If the above conditions are satisfied, then the Ingleton inequality is evidently violated. Conversely, let $A, B, C, D$ violate the Ingleton inequality. For each matroid $N$ with $A \cup B \cup C \cup D \subseteq E(N)$, let

$h_{1}(N)=r_{N}(A)+r_{N}(B)+r_{N}(A \cup B \cup D)+r_{N}(A \cup C \cup D)+r_{N}(C \cup D)$

and

$h_{2}(N)=r_{N}(A \cup B)+r_{N}(A \cup C)+r_{N}(A \cup D)+r_{N}(B \cup C)+r_{N}(B \cup D)$, so $h_{1}(M)>h_{2}(M)$ by assumption.

Claim 3.1.1. $A \cup B, A \cup C, A \cup D, B \cup C$ and $B \cup D$ are circuithyperplanes.

Proof of claim: Suppose otherwise. Let $M^{\prime}$ be obtained from $M$ by relaxing each circuit-hyperplane other than those among the five sets above, so $M^{\prime}$ is sparse paving and has at most four circuit-hyperplanes. 
By Lemma 2.2, $M^{\prime}$ is $\mathbb{R}$-representable, so $h_{1}\left(M^{\prime}\right) \leq h_{2}\left(M^{\prime}\right)$. By construction, we have $h_{2}\left(M^{\prime}\right)=h_{2}(M)$, and since $M^{\prime}$ is freer than $M$, we have $h_{1}\left(M^{\prime}\right) \geq h_{1}(M)$. Therefore

$$
h_{2}(M)<h_{1}(M) \leq h_{1}\left(M^{\prime}\right) \leq h_{2}\left(M^{\prime}\right)=h_{2}(M),
$$

a contradiction.

Claim 3.1.2. $|A|=|B|=r-2$, and the sets $A \cup B \cup C, A \cup B \cup D$ and $C \cup D$ are spanning in $M$.

Proof of claim: The first claim gives $h_{2}(M)=5 r-5$, so by assumption $h_{1}(M) \geq 5 r-4$. If $A \cup B \subseteq \mathrm{cl}_{M}(A)$, then we have

$h_{2}(M)=r_{M}(A)+r_{M}(A \cup B \cup C)+r_{M}(A \cup B \cup D)+r_{M}(B \cup C)+r_{M}(B \cup D)$

and $h_{2}(M)-h_{1}(M)=r_{M}(B \cup C)+r_{M}(B \cup D)-r_{M}(B)-r_{M}(C \cup D) \geq 0$ by submodularity, a contradiction. So $A \cup B \nsubseteq \mathrm{cl}_{M}(A)$; since $A \cup B$ is a circuit, it follows that $|A|=r_{M}(A) \leq r-2$ and, symmetrically, that $|B|=r_{M}(B) \leq r-2$. Therefore

$$
\begin{aligned}
3 r & \geq r_{M}(A \cup B \cup C)+r_{M}(A \cup B \cup D)+r_{M}(C \cup D) \\
& =h_{1}(M)-r_{M}(A)-r_{M}(B) \\
& \geq(5 r-4)-2(r-2)=3 r,
\end{aligned}
$$

so we have equality throughout, and $r_{M}(A \cup B \cup C)=r_{M}(A \cup B \cup D)=$ $r_{M}(C \cup D)=r$ while $|A|=|B|=r-2$.

For each nonempty subset $S$ of $\{A, B, C, D\}$, write $J_{S}$ for the collection of elements belonging to all sets in $S$ but no sets in $\{A, B, C, D\}-$ $S$, and let $n_{S}=\left|J_{S}\right|$. For example, $n_{A B}$ denotes $|(A \cap B)-(C \cup D)|$ (we omit commas and braces).

Since $A \cup C$ and $B \cup C$ are circuit-hyperplanes in a sparse paving matroid,

$$
\begin{aligned}
2 & \leq|(A \cup C)-(B \cup C)| \\
& =|A-(B \cup C)| \\
& =|A-B|-|(A \cap C)-B| \\
& =2-|(A \cap C)-B|,
\end{aligned}
$$

so $(A \cap C)-B=\varnothing$, giving $n_{A C}=n_{A C D}=0$. Using the symmetry between $A$ and $B$ and between $C$ and $D$, we also have $n_{A D}=n_{B C}=$ $n_{B D}=n_{B C D}=0$. Therefore $n_{C}+n_{C D}=n_{C}+n_{C D}+n_{B C D}+n_{B D}=$ $|C-A|=|C \cup A|-|A|=2$.

Since $A \cup C$ and $A \cup D$ are circuit-hyperplanes, we have $2 \leq \mid(A \cup$ $C)-(A \cup D) \mid=n_{D}+n_{B D}=n_{D}=2-n_{C D}$, from which we get $n_{C D}=0$ and $n_{D}=2$, and symmetrically $n_{C}=2$. Moreover $n_{A}=$ 
$n_{A}+n_{A C}+n_{A D}+n_{A C D}=|A-B|=|A \cup B|-|B|=r-(r-2)=2$, and symmetrically $n_{B}=2$.

The four undetermined $n_{S}$ thus far are $n_{A B}, n_{A B C D}, n_{A B C}$ and $n_{A B D}$; all others have been shown to be zero except $n_{A}=n_{B}=n_{C}=n_{D}=2$. Using the fact that $C \cup D$ is spanning, we thus have

$$
r \leq|C \cup D|=n_{A B C D}+n_{A B C}+n_{A B D}+n_{C}+n_{D} .
$$

On the other hand,

$$
r-2=|A|=n_{A B C D}+n_{A B C}+n_{A B D}+n_{A B}+n_{A}
$$

since $n_{A}=n_{C}=n_{D}=2$, these together imply that $n_{A B}=0$. The above also gives $n_{A B C D}+n_{A B C}+n_{A B D}=r-4$. Now setting $\left(X_{1}, X_{2}, X_{3}, X_{4}, Y, Z_{1}, Z_{2}\right)=\left(J_{A}, J_{B}, J_{C}, J_{D}, J_{A B C D}, J_{A B C}, J_{A B D}\right)$ gives the required structure. Finally, we see that $|C \cup D|=n_{A B C D}+n_{A B D}+$ $n_{A B C}+n_{C}+n_{D}=(r-4)+4=r$; since $C \cup D$ is spanning, it must be a basis.

A simpler characterisation of these matroids below follows with $K=$ $Z \cup Y_{1} \cup Y_{2}$ and the $P_{i}$ equal to some ordering of the $X_{i}$ above.

Corollary 3.2. Let $M$ be a sparse paving matroid. Then $M$ is nonIngleton if and only if there are pairwise disjoint sets $P_{1}, P_{2}, P_{3}, P_{4}, K$ so that $|K|=r-4$ and $\left|P_{i}\right|=2$ for each $i$, and exactly five of the six sets of the form $K \cup P_{i} \cup P_{j}: i \neq j$ are circuit-hyperplanes of $M$.

As observed in Cam13, If $M$ is a matroid for which the above condition holds, then it also holds in the eight-element, rank-4 matroid $(M / K) \mid\left(P_{1} \cup P_{2} \cup P_{3} \cup P_{4}\right)$; therefore, every non-Ingleton sparse paving matroid has an eight-element, rank-4 non-Ingleton sparse paving matroid as a minor. Mayhew and Royle [MR08] showed that there are precisely 39 such matroids; for every such matroid $N$, the Vámos matroid $V_{8}$ can be obtained from $N$ by a sequence of circuit-hyperplane relaxations. (We remark that MR08 uses different terminology from ours, calling these matroids 'Ingleton non-representable' rather than 'nonIngleton'.) The unique minor-minimal matroids that are not sparse paving are $U_{0,2} \oplus U_{1,1}$ and $U_{2,2} \oplus U_{0,1}$; together these facts imply Theorem 1.3 .

\section{Counting Ingleton Matroids}

The proof of the following theorem uses techniques from CM14, Proposition 2.1]. 
Theorem 4.1. There exists $n_{0}$ such that for all $n \geq n_{0}$ and all $0<$ $r<n$, the number of rank-r Ingleton matroids with ground set $[n]$ is at

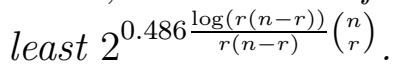

Proof. We may assume that $2 \leq r \leq \frac{n}{2}$, since otherwise the theorem is trivial or follows by duality. Write $N=\left(\begin{array}{l}n \\ r\end{array}\right)$ and $d=r(n-r)$ for the number of vertices and the valency of the Johnson graph $J(n, r)$.

For $x \in \mathbb{R}$, let $f(x)=1-\frac{1}{2} x-\frac{1}{64} x^{4}$. We show that if $c>0$ is a real number and $\gamma<c f(c)$, then there are at least $2^{\gamma \frac{\log d}{d} N}$ Ingleton sparse paving matroids of rank $r$ on $[n]$, provided $n$ is sufficiently large; the result as stated follows with $c=0.95$ and $\gamma=0.486$.

Given $c$ and $\gamma$, let $\alpha$ be such that $\gamma / c<\alpha<f(c)$ and let $\epsilon=f(c)-\alpha$, so $1-f(c)+\epsilon=1-\alpha$. Set $k=\left\lfloor c \frac{N}{d}\right\rfloor$, so $(1-o(1)) \frac{c}{d} \leq \frac{k}{N} \leq \frac{c}{d}$. Pick a $k$-set $H$ of vertices in $J(n, r)$ uniformly at random from among all $k$-subsets of vertices and write $E(H)$ for the set of unordered pairs of vertices in $H$ joined by an edge in $J(n, r)$, and $e_{H}$ for $|E(H)|$.

Claim 4.1.1. $\mathbf{E}\left(e_{H}\right) \leq \frac{c k}{2}$ and $\operatorname{Var}\left(e_{H}\right)=o\left(k^{2}\right)$.

Proof of claim: We have

$$
\mathbf{E}\left(e_{H}\right)=\frac{1}{2} d N \frac{\left(\begin{array}{c}
N-2 \\
k-2
\end{array}\right)}{\left(\begin{array}{l}
N \\
k
\end{array}\right)} \leq \frac{d k^{2}}{2 N} \leq \frac{c k}{2} .
$$

Let $\Theta$ denote the set of ordered pairs $(e, f)$ of edges of $J(n, r)$. Write $\Theta_{j}$, $j \in\{0,1,2\}$, for the set of pairs in $\Theta$ that span $4-j$ vertices. Note that $|\Theta|=\frac{1}{4} d^{2} N^{2}$, while $\left|\Theta_{1}\right|=N d(d-1) \leq N d^{2}$ and $\left|\Theta_{2}\right|=\frac{1}{2} d N$. Now, using the fact that $\left(\begin{array}{l}N-\ell \\ k-\ell\end{array}\right) /\left(\begin{array}{l}N \\ k\end{array}\right)=(1+o(1))(k / N)^{\ell}$ for each constant $\ell$, we have

$$
\begin{aligned}
\operatorname{Var}\left(e_{H}\right)= & \sum_{(e, f) \in \Theta}[\operatorname{Pr}(e, f \in E(H))-\operatorname{Pr}(e \in E(H)) \operatorname{Pr}(f \in E(H))] \\
= & \left|\Theta_{0}\right|\left(\frac{\left(\begin{array}{l}
N-4 \\
k-4
\end{array}\right)}{\left(\begin{array}{l}
N \\
k
\end{array}\right)}-\frac{\left(\begin{array}{l}
N-2 \\
k-2
\end{array}\right)^{2}}{\left(\begin{array}{l}
N \\
k
\end{array}\right)^{2}}\right)+\left|\Theta_{1}\right|\left(\frac{\left(\begin{array}{l}
N-3 \\
k-3
\end{array}\right)}{\left(\begin{array}{l}
N \\
k
\end{array}\right)}-\frac{\left(\begin{array}{l}
N-2 \\
k-2
\end{array}\right)^{2}}{\left(\begin{array}{l}
N \\
k
\end{array}\right)^{2}}\right) \\
& \quad+\left|\Theta_{2}\right|\left(\frac{\left(\begin{array}{c}
N-2 \\
k-2
\end{array}\right)}{\left(\begin{array}{c}
N \\
k
\end{array}\right)}-\frac{\left(\begin{array}{l}
N-2 \\
k-2
\end{array}\right)^{2}}{\left(\begin{array}{l}
N \\
k
\end{array}\right)^{2}}\right) \\
\leq & \frac{1}{4} N^{2} d^{2} O\left(\frac{k^{4}}{N^{4}}\right)+N d^{2}\left(\frac{k}{N}\right)^{3}+\frac{1}{2} d N\left(\frac{k}{N}\right)^{2} \\
= & o\left(d^{-2} N^{2}\right)+O\left(d^{-1} N\right)+O\left(d^{-1} N\right)=o\left(k^{2}\right),
\end{aligned}
$$

since $k=(1+o(1)) c N / d$. 
Let $\Omega$ be the set of all pairs $\left(\left\{P_{1}, P_{2}, P_{3}, P_{4}\right\}, K\right)$ where $P_{1}, P_{2}, P_{3}, P_{4}, K$ are pairwise disjoint subsets of $[n]$ with $\left|P_{i}\right|=2$ and $|K|=r-4$ (note that the collection of $P_{i}$ is unordered). Now

$$
\begin{aligned}
|\Omega| & =\frac{1}{4 !}\left(\begin{array}{c}
8 \\
2,2,2,2
\end{array}\right)\left(\begin{array}{l}
n \\
8
\end{array}\right)\left(\begin{array}{l}
n-8 \\
r-4
\end{array}\right) \\
& =\frac{8 !}{2^{4} \cdot 4 !}\left(\begin{array}{l}
n \\
r
\end{array}\right) \frac{r !(n-r) !}{8 !(r-4) !(n-r-4) !} \\
& \leq \frac{d^{4}}{2^{7} \cdot 3} N .
\end{aligned}
$$

For each $\omega \in \Omega$, let $U(\omega)=\left\{K \cup P_{i} \cup P_{j}:\{i, j\} \in\left(\begin{array}{c}{[4]} \\ 2\end{array}\right)\right\}$, so $|U(\omega)|=$ 6. For each $H$ and each $i \in\{0, \ldots, 6\}$, let $b_{i, H}$ denote the number of $\omega$ in $\Omega$ for which $|H \cap U(\omega)|=i$.

Claim 4.1.2. $\mathbf{E}\left(b_{5, H}\right) \leq \frac{c^{4} k}{64}$ and $\mathbf{E}\left(b_{6, H}\right)=o(k)$ while $\operatorname{Var}\left(b_{5, H}\right)=$ $o\left(k^{2}\right)$.

Proof of claim: The claim is trivial when $r<4$ since $\Omega$ is empty, so suppose that $r \geq 4$. Given $\omega \in \Omega$, the probability that $|H \cap U(\omega)|=i$ is $\left(\begin{array}{c}6 \\ i\end{array}\right)\left(\begin{array}{c}N-6 \\ k-i\end{array}\right) /\left(\begin{array}{c}N \\ k\end{array}\right) \leq\left(\begin{array}{c}6 \\ i\end{array}\right)(k / N)^{i} \leq\left(\begin{array}{l}6 \\ i\end{array}\right) c^{i} d^{-i}$, so

$$
\mathbf{E}\left(b_{i, H}\right) \leq|\Omega|\left(\begin{array}{l}
6 \\
i
\end{array}\right) c^{i} d^{-i} \leq\left(\begin{array}{l}
6 \\
i
\end{array}\right) \frac{c^{i} d^{4-i}}{2^{7} \cdot 3} N \leq\left(\begin{array}{l}
6 \\
i
\end{array}\right) \frac{c^{i-1} d^{5-i} k}{2^{7} \cdot 3},
$$

giving $\mathbf{E}\left(b_{5, H}\right) \leq \frac{c^{4} k}{64}$ and $\mathbf{E}\left(b_{6, H}\right)=o(k)$ as required.

Let $\Pi=\Omega^{2}$, so $|\Pi|=|\Omega|^{2} \leq d^{8} N^{2}$. Let $\Pi_{0}:=\{(\omega, \omega): \omega \in \Omega\} \subseteq \Pi$, let $\Pi_{2}$ be the set of all $\left(\omega_{1}, \omega_{2}\right) \in \Pi$ for which $U\left(\omega_{1}\right) \cap U\left(\omega_{2}\right)=\emptyset$, and let $\Pi_{1}=\Pi \backslash\left(\Pi_{0} \cup \Pi_{2}\right)$. Since $U(\omega)$ contains 6 vertices of $J(n, r)$ for each $\omega \in \Omega$, symmetry and a counting argument gives that for each vertex $v$ of $J(n, r)$, we have

$$
|\{\omega \in \Omega: v \in U(\omega)\}|=\frac{6|\Omega|}{N}=O\left(d^{4}\right) .
$$

It follows that $\left|\Pi_{1}\right|=O\left(d^{8} N\right)$. Call an $\omega \in \Omega$ bad for $H$ if $|H \cap U(\omega)|=$ 5. Recall that the probability that a given $\omega$ is bad is $6\left(\begin{array}{l}N-6 \\ k-5\end{array}\right) /\left(\begin{array}{l}N \\ k\end{array}\right)=$ $(6+o(1)) k^{5} / N^{5}$. Note that $\omega$ is determined uniquely by any four sets in $U(\omega)$; it follows that if $\left(\omega_{1}, \omega_{2}\right) \in \Pi_{1}$ then $U\left(\omega_{1}\right)$ and $U\left(\omega_{2}\right)$ have at most three sets in common, so if both $\omega_{1}$ and $\omega_{2}$ are bad, then $H$ contains at least seven of the sets in $U\left(\omega_{1}\right)$ and $U\left(\omega_{2}\right)$. Since $\left|U\left(\omega_{1}\right) \cup U\left(\omega_{2}\right)\right| \leq 10$, a pair $\left(\omega_{1}, \omega_{2}\right) \in \Pi_{1}$ is thus bad with probability at most $\left(\begin{array}{c}10 \\ 7\end{array}\right)\left(\begin{array}{c}N-7 \\ k-7\end{array}\right) /\left(\begin{array}{c}N \\ k\end{array}\right) \leq\left(\begin{array}{c}10 \\ 7\end{array}\right)(k / N)^{7}=O\left(d^{-7}\right)$. If $\left(\omega_{1}, \omega_{2}\right) \in \Pi_{0}$ then $\omega_{1}$ and $\omega_{2}$ are both bad with probability $(6+o(1)) k^{5} / N^{5}=O\left(d^{-5}\right)$. 
If $\left(\omega_{1}, \omega_{2}\right) \in \Pi_{2}$ then both are bad with probability $36\left(\begin{array}{c}N-12 \\ k-10\end{array}\right) /\left(\begin{array}{l}N \\ k\end{array}\right)=$ $(36+o(1)) k^{10} / N^{10}$. Therefore

$$
\begin{aligned}
\operatorname{Var}\left(b_{5, H}\right)= & \sum_{\left(\omega_{1}, \omega_{2}\right) \in \Pi}\left[\operatorname{Pr}\left(\omega_{1}, \omega_{2} \text { bad }\right)-\operatorname{Pr}\left(\omega_{1} \text { bad }\right) \operatorname{Pr}\left(\omega_{2} \text { bad }\right)\right] \\
\leq & \left|\Pi_{2}\right|\left(\frac{(36+o(1)) k^{10}}{N^{10}}-\left(\frac{(6+o(1)) k^{5}}{N^{5}}\right)^{2}\right)+\left|\Pi_{0}\right| O\left(d^{-5}\right) \\
& \quad+\left|\Pi_{1}\right| O\left(d^{-7}\right) \\
\leq & d^{8} N^{2} o\left(k^{10} / N^{10}\right)+O\left(d^{-1} N\right)+O(d N) \\
= & o\left(d^{-2} N^{2}\right)+O(d N) .
\end{aligned}
$$

Now $d^{-2} N^{2}=(1+o(1)) k^{2}$, and, using $4 \leq r \leq \frac{n}{2}$, we have $d N=r(n-$ $r)\left(\begin{array}{l}N \\ r\end{array}\right)=o\left(\frac{1}{r^{2}(n-r)^{2}}\left(\begin{array}{l}N \\ r\end{array}\right)^{2}\right)=o\left(k^{2}\right)$. It follows that $\operatorname{Var}\left(b_{5, H}\right)=o\left(k^{2}\right)$ as required.

By the two claims, the random variables $e_{H}$ and $b_{5, H}$ have means at most $\frac{c k}{2}$ and $\frac{c^{4} k}{64}$ respectively, and both have standard deviations in $o(k)$; it follows by Chebyshev's inequality that $\operatorname{Pr}\left(e_{H}>\left(\frac{c}{2}+\frac{\epsilon}{3}\right) k\right)=o(1)$ and $\operatorname{Pr}\left(b_{5, H}>\left(\frac{c^{4}}{64}+\frac{\epsilon}{3}\right) k\right)=o(1)$. Since $\mathbf{E}\left(b_{6, H}\right) \in o(k)$, Markov's inequality gives $\operatorname{Pr}\left(b_{6, H}>\frac{\epsilon}{6} k\right)=o(1)$. Therefore, with probability $1-o(1)$, we have

$$
e_{H}+b_{5, H}+2 b_{6, H} \leq\left(\frac{c}{2}+\frac{c^{4}}{64}+\epsilon\right) k=(1-f(c)+\epsilon) k=(1-\alpha) k .
$$

Call a set $W \subseteq\left(\begin{array}{c}{[n]} \\ r\end{array}\right)$ good if $e_{W}=b_{5, W}=b_{6, W}=0$. Each set $H \subseteq\left(\begin{array}{c}{[n]} \\ r\end{array}\right)$ of size $k$ contains a good set $W$ of size $|H|-e_{H}-b_{5, H}-2 b_{6, H}$. With probability $1-o(1)$ we have $e_{H}+b_{5, H}+2 b_{6, H} \leq(1-\alpha) k$ and so $|W| \geq k-(1-\alpha) k=\alpha k$; thus there are at least $(1-o(1))\left(\begin{array}{l}N \\ k\end{array}\right)$ different choices of $H$ that contain a good set $W$ of size at least $\alpha k$. On the other hand, each good set $W$ of size at least $\alpha k$ is contained in at most $\left(\begin{array}{c}N \\ (1-\alpha) k\end{array}\right)$ different $H$; therefore the number of good sets is at least $\nu=(1-o(1))\left(\begin{array}{l}N \\ k\end{array}\right) /\left(\begin{array}{c}N \\ (1-\alpha) k\end{array}\right)$. We have

$$
\begin{aligned}
\log \nu & =\log \left(\begin{array}{c}
N \\
k
\end{array}\right)-\log \left(\begin{array}{c}
N \\
(1-\alpha) k
\end{array}\right)-o(1) \\
& \geq k \log (N / k)-k \log (e N /(1-\alpha) k)-o(1) \\
& =(1-o(1)) \alpha k \log (N / k) \\
& =(1-o(1)) \alpha c \frac{\log d}{d} N .
\end{aligned}
$$

But for large $n$ we have $(1-o(1)) \alpha c>\gamma$, so there are at least $2^{\gamma \frac{\log d}{d} N}$ good stable sets. By Corollary 3.2 , each such set is the collection of 
circuit-hyperplanes of an Ingleton sparse paving matroid of rank $r$ on ground set $[n]$; the theorem follows.

We have attempted to optimize the constant $0.486 \ldots$ in the exponent as much as possible within the constraints of our techniques; the proof can be simplified to use first rather than second moments, at the expense of a lowered constant of around 0.4. One case where the constant can certainly be improved is where the rank $r$ (or, dually, the corank $n-r)$ is constant, in which case the estimate on $|\Omega|$ can be improved by an asymptotically significant factor of $\left(1-\frac{1}{r}\right)\left(1-\frac{2}{r}\right)\left(1-\frac{3}{r}\right)$. Carrying through this better estimate has the effect of slightly increasing the constant in the exponent further towards 0.5 , giving 0.5 exactly when $r \leq 3$, and roughly 0.498 when $r=4$.

We complement the above enumeration result, which is based on the construction of a large family of sparse paving matroids each of which contain roughly $\frac{1}{r(n-r)}\left(\begin{array}{l}n \\ r\end{array}\right)$ circuit-hyperplanes, by a construction that shows that sparse paving Ingleton matroids with many more circuithyperplanes exist.

We sketch the construction, which is originally due to Graham and Sloane [GS80]. Suppose that $0<r<n$. The function $c: V(J(n, r)) \rightarrow$ $\mathbb{Z}_{n}$ given by $c(X)=\sum_{x \in X} x \bmod n$ is a proper vertex colouring of $J(n, r)$. It follows that for each $\gamma \in \mathbb{Z}_{n}$, the set $c^{-1}(\gamma) \equiv\{X: c(X)=$ $\gamma\}$ is a stable set in $J(n, r)$ and hence $S(n, r, \gamma):=\left([n],\left(\begin{array}{c}{[n]} \\ r\end{array}\right) \backslash c^{-1}(\gamma)\right)$ is the sparse paving matroid whose set of circuit-hyperplanes is $c^{-1}(\gamma)$.

Lemma 4.2. $S(n, r, \gamma)$ is Ingleton.

Proof. For the sake of contradiction, suppose that $A, B, C, D \subseteq[n]$ violate Ingleton's inequality and obtain $K, P_{1}, P_{2}, P_{3}, P_{4}$ as in Corollary [3.2. Write $P_{i}=\left\{p_{i}, p_{i}^{\prime}\right\}$. We may assume that $K \cup P_{i} \cup P_{j}$ is a circuit-hyperplane for all $\{i, j\} \in\left(\begin{array}{c}{[4]} \\ 2\end{array}\right) \backslash\{\{3,4\}\}$, while $K \cup P_{3} \cup P_{4}$ is a basis. Define $\gamma^{\prime}=\gamma-\sum_{x \in K} x \bmod n$. It follows that

$$
p_{1}+p_{1}^{\prime}+p_{2}+p_{2}^{\prime}=p_{1}+p_{1}^{\prime}+p_{3}+p_{3}^{\prime}=p_{2}+p_{2}^{\prime}+p_{4}+p_{4}^{\prime}=\gamma^{\prime},
$$

so in particular

$$
p_{3}+p_{3}^{\prime}+p_{4}+p_{4}^{\prime}=\gamma^{\prime},
$$

which implies that $c\left(K \cup P_{3} \cup P_{4}\right)=\gamma$, contradicting that $K \cup P_{3} \cup P_{4}$ is a basis of $S(n, r, \gamma)$.

Corollary 4.3. For all $0<r<n$, there exists a sparse paving Ingleton matroid of rank $r$ on ground set $[n]$ that has at least $\frac{1}{n}\left(\begin{array}{l}n \\ r\end{array}\right)$ circuithyperplanes. 
Proof. As $\left\{c^{-1}(\gamma): \gamma \in \mathbb{Z}_{n}\right\}$ partitions $V(J(n, r))$, there is $\gamma_{0} \in \mathbb{Z}_{n}$ such that $\left|c^{-1}\left(\gamma_{0}\right)\right| \geq \frac{1}{n}\left(\begin{array}{l}n \\ r\end{array}\right)$. Consequently, the matroid $S\left(n, r, \gamma_{0}\right)$, which is Ingleton by Lemma 4.2, has at least $\frac{1}{n}\left(\begin{array}{l}n \\ r\end{array}\right)$ circuit-hyperplanes.

\section{REFERENCES}

[BPvdP15] N. Bansal, R.A. Pendavingh, and J.G. van der Pol. On the number of matroids. Combinatorica, 35(3):253-277, 2015.

[Cam13] Amanda Cameron. Kinser inequalities and related matroids. Master's thesis, Victoria University of Wellington, 2013. Available from arXiv:1401.0500.

[CM14] Jeff Cooper and Dhruv Mubayi. Counting independent sets in trianglefree graphs. Proceedings of the American Mathematical Society, 142(10):3325-3334, 2014.

[GS80] R.L Graham and N.J.A. Sloane. Lower bounds for constant weight codes. IEEE Transactions on Information Theory, 26(1), 1980.

[Ing71] A.W. Ingleton. Representation of matroids. In D.J.A. Welsh, editor, Combinatorial mathematics and its applications (Proceedings of a conference held at the Mathematical Institute, Oxford, from 7-10 July, 1969). Academic Press, 1971.

[MNW09] Dillon Mayhew, Mike Newman, and Geoff Whittle. On excluded minors for real-representability. Journal of Combinatorial Theory, Series $B, 99: 685-689,2009$.

[MNWW11] Dillon Mayhew, Mike Newman, Dominic Welsh, and Geoff Whittle. On the asymptotic proportion of connected matroids. European Journal of Combinatorics, 32(6):882-890, 2011.

[MR08] Dillon Mayhew and Gordon F. Royle. Matroids with nine elements. Journal of Combinatorial Theory, Series B, 98(2):415-431, 2008.

[Nel16] Peter Nelson. Almost all matroids are non-representable. Preprint, available from arXiv:1605.04288v2, 2016.

[Oxl11] James Oxley. Matroid theory, volume 21 of Oxford graduate texts in mathematics. Oxford University Press, second edition, 2011.

Department of Combinatorics and Optimization, University of WAterloo, Waterloo, Canada

Department of Mathematics and Computer Science, Eindhoven UniVersity of Technology, Eindhoven, The Netherlands 